\title{
Electronic Clinical Challenges and Images in GI
}

\section{A Rare Cause of Abdominal Pain}

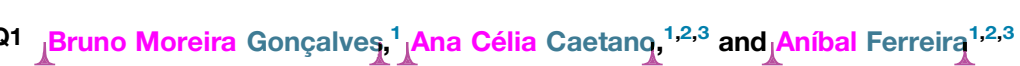

${ }^{1}$ Department of Gastroenterology, Hospital Braga, ${ }^{2}$ Life and Health Sciences Research Institute (ICVS), School of Health Sciences, University of Minho, Braga, and ${ }^{3}$ ICVS/3B's - PT Government Associate Laboratory, Braga/Guimarães, Portugal
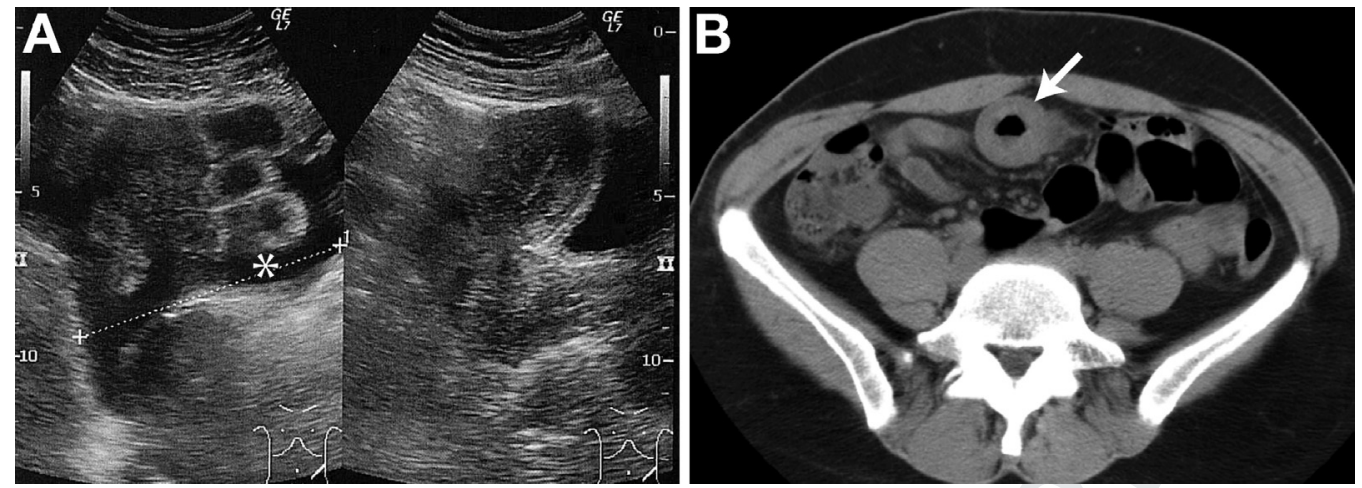

Question: A 47-year-old woman presented to the emergency room with acute diffuse abdominal pain and emesis. The patient's medical history included hypertension for which she started lisinopril $20 \mathrm{mg} 3$ days before. On our observation, the vital signs were normal and the abdominal examination revealed periumbilical tenderness, without signs of peritonitis. Laboratory analysis was notable only for a slightly elevated C-reactive protein (6.45 $\mathrm{mg} / \mathrm{L}$ ). Abdominal ultrasonography (Figure $A$ ) and computed tomography (Figure $B$ ) were taken.

What is the diagnosis?

See the GaStroenterology web site (www.gastrojournal.org) for more information on submitting your favorite image to Clinical Challenges and Images in GI.

Acknowledgments: The authors acknowledge the contribution and critical review of the manuscript by Dr Pedro Bastos ${ }^{1}$ and Prof Carla Rolanda. ${ }^{1,2,3}$

Conflicts of interest: The authors disclose no conflicts.

(c) 2013 by the AGA Institute

0016-5085/\$36.00

http://dx.doi.org/10.1053/j.gastro.2013.07.002 


\section{Electronic Clinical Challenges and Images in GI, contimued}

Answer to the Clinical Challenges and Images in GI Question: Image 6: AngiotensinConverting Enzyme Inhibitor-Induced Visceral Angioedema

Abdominal ultrasonography revealed a small amount of ascites (Figure $A$, star) and computed tomography showed a circumferential jejunal wall thickening (Figure B, arrow), prominent mesenteric vessels and lymphadenopathy. Serum levels of C1 and C4 were normal. A diagnosis of isolated visceral angioedema owing to angiotensin-converting enzyme (ACE) inhibitor was made. The patient suspended lisinopril with resolution of signs and symptoms within 36 hours.

ACE inhibitors are widely used to control hypertension and $>40$ million patients are currently taking ACE inhibitors or angiotensin-receptor blocker. ${ }^{1}$ Angioedema can affect $0.1 \%-0.7 \%$ of patients taking this medication, mostly during the first week of therapy and with peripheral manifestations (swelling of the face, tongue, and lips). It results from vasodilatation, which leads to serum accumulation in the interstitial space. Visceral angioedema presents a diagnostic challenge requiring a high level of suspicion. As with the present case, most present in women within 72 hours of commencing therapy as abdominal pain or ascites. ${ }^{2}$ Small bowel wall abnormalities (thickening, dilation and straightening) are common. ${ }^{3}$

In most patients, symptoms resolved within 2-4 days of stopping the ACE inhibitor. Angioedema can recur in up to one third of patients who switch from an ACE inhibitor to an angiotensin-receptor blocker; thus, both therapies should be avoided in these cases.

\section{References}

1. Agostoni A, Cicardi M. Drug-induced angioedema without urticaria. Drug Saf 2001;24:599-606.

2. Korniyenko A, Alviar CL, Cordova JP, et al. Visceral angioedema due to angiotensin-converting enzyme inhibitor therapy. Cleve Clin J Med 2011;78:297-304.

3. Scheirey CD, Scholz FJ, Shortsleeve MJ, et al. Angiotensin-converting enzyme inhibitor-induced small-bowel angioedema: clinical and imaging findings in 20 patients. AJR Am J Roentgenol 2011;197:393-398. 